\title{
Pacifismo, antimilitarismo y libertad de expresión en Madrid (1984-1990): Radio Cero, la radio anti-OTAN
}

\author{
Bakezaletasuna, antimilitarismoa eta adierazpen askatasuna Madrilen \\ (1984-1990): Radio Cero, NATOren aurkako irratia
}

Pacifism, antimilitarism and freedom of speech in Madrid (1984-1990): Radio Cero, the anti-NATO radio

José Emilio Pérez Martínez ${ }^{\star}$

Université Paris-Sorbonne (Paris IV)

RESUMEN: Durante la década de 1980 la lucha contra la OTAN fue una de las principales movilizaciones sociales en España. A partir de la victoria electoral del PSOE en 1982, la intensidad de la lucha creció, sumando a nuevos actores. Entre ellos estaban la Comisión Anti-OTAN y Radio Cero, una radio libre. A esta última, y a cómo se relacionaron en su praxis la lucha antimilitarista y por la libertad de expresión, le dedicaremos estas páginas viendo su desarrollo, cómo implementó esas luchas y cómo se convirtió en un medio de proyección ideológica que puso estas movilizaciones en la esfera pública.

PALABRAS CLAVE: Radio, movimientos sociales, comunicación social, libertad de expresión.

ABSTRACT: During the decade of 1980 the struggle against the NATO was one of the main social mobilizations in Spain. Right after the victory of the PSOE in 1982 elections, the intensity of the struggle grew, adding new actors to the mobilization. Among them were the Comision Anti-OTAN and Radio Cero, a free radio. In these pages we will attend to this radio station, and to how the antimilitarist and the freedom of speech struggles were linked in its daily praxis, seeing its development, how it implemented those fights and how it became a mean of ideological projection which put all those mobilizations in the public sphere.

KEYWORDS: Radio, social movements, social communication, freedom of speech.

\footnotetext{
* Correspondencia a / Corresponding author: José Emilio Pérez Martínez. Université Paris-Sorbonne (Paris IV), Centre malesherbes, 108 Bv. Malesherbes, 3. étage (75017 París) - joseempe@ucm.es - https://orcid.org/0000-0003-1880-5424

Cómo citar / How to cite: Pérez Martínez, José Emilio (2019). «Pacifismo, antimilitarismo y libertad de expresión en Madrid (1984-1990): Radio Cero, la radio anti-OTAN", Zer, 25(47), 85-104. (https://doi.org/10.1387/zer.20773).

Recibido: 23 abril, 2019; aceptado: 6 septiembre, 2019.

ISSN 1137-1102 - eISSN 1989-631X / (c) 2019 UPV/EHU

(c) (7) Esta obra está bajo una licencia

Creative Commons Atribución 4.0 Internacional
} 


\section{Introducción}

Tradicionalmente se considera el referéndum sobre la permanencia de España en la Organización del Tratado del Atlántico Norte (OTAN), y su correspondiente campaña, el canto de cisne de la izquierda española de la Transición. Pero antes de que esto sucediera, la movilización contra la Alianza Atlántica fue uno de los principales caballos de batalla de la izquierda parlamentaria, la radical y los movimientos sociales.

Los primeros gobiernos del tránsito a la democracia, de la Unión de Centro Democrático (UCD), iniciaron la incorporación de España a la misma. Un proceso marcado, primero, por la indecisión y la ambigüedad durante los mandatos de Suárez, que centró su política exterior en el mundo árabe y en América Latina (Powell, 2003: 102), y que se concretó durante el gobierno de Leopoldo Calvo Sotelo, cuya postura era abiertamente "atlantista». Olvidando las promesas de un debate amplio sobre la cuestión (Mesa, 1982: 38) y rompiendo con su programa electoral (Aldecoa, 1989: 72), se comunicó a la Alianza su voluntad de adhesión a finales del año 1981, y en mayo de 1982 España se convirtió en el miembro número 16 de esta estructura militar. Una incorporación que se hizo con la negativa de parte de la oposición parlamentaria, el Partido Socialista Obrero Español (PSOE) y el Partido Comunista de España (PCE), de la izquierda radical y de los movimientos sociales.

Las elecciones generales de octubre de 1982 las ganó el PSOE, partido que defendía aquello de "OTAN, de entrada, no» y que llevaba en su programa la convocatoria de un referéndum sobre la permanencia del país en esta organización, comenzando así un periodo de reflexión que llevó «a la suspensión de las conversaciones sobre la integración militar española en la OTAN» (Ministerio, 2017).

El ejecutivo socialista de Felipe González cambió sus posiciones con respecto a la relación entre España y la OTAN hacia 1984, entendiendo que la permanencia favorecía el proyecto modernizador del gobierno y evitaba el aislamiento internacional del país, lo que causó que la intensidad de las movilizaciones creciera y se comenzara a reclamar la convocatoria del prometido referéndum. Puede entenderse que:

la llegada al poder del PSOE trajo consigo una cura de realismo político, convirtiéndose las cuestiones de defensa y seguridad en uno de los últimos ajustes ideológicos de los socialistas, pese a la existencia de un potente movimiento pacifista y de un terror ciudadano a un posible holocausto nuclear, consecuencia de una guerra entre bloques. (Mateos, 2016: 14)

Una vez anunciada la fecha del referéndum, convocado para 1986, los agentes implicados en la lucha anti-OTAN crecieron exponencialmente. Uno de ellos será el protagonista de estas páginas: las radios libres. 
A finales de la década de 1970 surgieron en España los primeros proyectos de este tipo, emisoras pequeñas, precarias en lo técnico, pero con una clara vocación de renovación del panorama comunicativo del momento. Estas radios se desarrollaron durante la década de 1980, hasta que en 1989 se vieron obligadas a suspender temporalmente sus emisiones para poder participar en un concurso de licencias vinculado a la Ley de Ordenación de las Telecomunicaciones (LOT), produciéndose el primer punto de inflexión en su historia.

Unas emisoras que dieron cabida en sus parrillas a los movimientos sociales, poniéndose, como herramienta de comunicación, a su servicio y dando voz a aquellos sectores que no la tenían, asegurando el derecho de acceso a los medios de comunicación social. Se atenderá aquí al caso concreto de Radio Cero, emisora madrileña vinculada con la lucha contra la OTAN, el pacifismo y el antimilitarismo. Se verá su desarrollo, su funcionamiento, sus principios y cómo articuló las luchas pacifista y antimilitarista con las reivindicaciones propias de las radios libres. Para ello nos apoyaremos en las escasa fuentes recuperadas — entrevistas personales a miembros de la emisora y documentación de la misma - y en la prensa, con el fin de intentar reconstruir cómo esta radio llevó a cabo dicha articulación y ver qué significado tuvo dentro de la propia emisora y para el movimiento anti-OTAN. Comenzaremos dando un necesario contexto sobre la evolución de estos dos movimientos sociales (anti-OTAN y radios libres), como punto de partida para este análisis.

\section{Unas notas sobre el movimiento pacifista en Madrid y la Comisión Anti-OTAN}

Debe tenerse en cuenta aquello que ya señalara Pablo Socorro Arencibia: «sobre el movimiento anti-OTAN a nivel estatal no existe, a día de hoy, un estudio histórico pormenorizado que estudie sus implicaciones y recorrido durante los años ochenta» (Socorro, 2005: 11), aunque sí existen algunas aproximaciones locales (Gonzalo, 2011; Fernández Amador \& Vidal Gómez, 2004; o Wilhelmi Casanova, 2011). Un hecho que, por un lado, dificulta una comprensión global del movimiento y que, por otro, justifica la pertinencia de trabajos como este que ayudan a avanzar en su conocimiento.

El movimiento anti-OTAN constituye una de las ramificaciones del antimilitarismo y el pacifismo. Tiene su origen en la respuesta al progresivo acercamiento que tienen algunos líderes de la UCD a la idea de incorporar el país a la Alianza. Un movimiento que comienza a gestarse lentamente en torno a 1979 y que hasta 1982 no puede entenderse como una realidad estructurada, en la que una de las distintas ramas fue dinamizada principalmente por dos partidos de la izquierda radical, el Movimiento Comunista (MC) y la Liga Comunista Revolucionaria (LCR). 
En Madrid existía ya un tejido pacifista (Oliver, 2011) conformado por activistas provenientes del movimiento de objeción de conciencia y del ámbito de la noviolencia, que adquirió con la incorporación de los militantes de estos dos partidos, un nuevo cariz antiimperialista, pues las reivindicaciones contra la OTAN estaban impregnadas de un rechazo a las políticas estadounidenses. Una lucha que da su primer paso relevante el 25 de enero de 1981 con la primera Marcha a Torrejón, una manifestación desde Madrid a la base americana situada en dicha localidad, a unos 12 kilómetros de la capital. Una movilización que recabó apoyo de diversos sectores de la sociedad civil organizada, como el movimiento ecologista, y que con su éxito hizo que los primeros Comités anti-OTAN de los barrios de Madrid comenzaran a expandir su influencia, encuadrando a cada vez más vecinos y repitiéndose la manifestación en otras ocasiones (Mercado, 1982; García, 1985; García 1986b ). Este impulso se vio frenado por la intentona golpista del 23 de febrero de 1981, que generó miedos entre los militantes de la izquierda radical, haciendo que no se retomara la lucha hasta avanzado el año. De acuerdo con Carlos Otamendi, «en un intento de borrar las sombras del golpe y de seguir batallando contra la OTAN» (El País, 1986) se recuperó la actividad de los Comités, se organizó en la Casa de Campo de Madrid un festival contra la Alianza al que acudieron 50.000 personas, y comenzó a perfilarse, hacia 1982, la Comisión anti-OTAN (CAO), que terminará por convertirse en un organismo de coordinación de las movilizaciones en este campo con la intención de englobar «el mayor número posible de sensibilidades pacifistas y antimilitaristas» (Socorro Arencibia, 2015: 54). La CAO, con un fuerte impulso del MC y de la LCR, se convirtió en un espacio de referencia, pretendidamente apartidista, con la participación de importantes figuras como Carlos Otamendi (MC), y Manuel Garí (LCR) e independientes. La Comisión conformó un particular acercamiento a las posiciones pacifistas, con sus propias aportaciones teóricas y agenda, que la enfrentarían a otras propuestas como las organizadas por el PCE, la Asociación por la Paz y el Desarme (APD) y el PSOE, el Movimiento por la Paz, el Desarme y la Libertad $(\mathrm{MPDL})^{1}$.

Con la llegada del PSOE al gobierno en el año 1982 y el progresivo cambio de sus posiciones, el movimiento contra la Alianza Atlántica se reorganiza y en 1983 se crea la Coordinadora Estatal de Organizaciones Pacifistas (CEOP), en la sede de la $\mathrm{CAO}$, «en un intento de unificar a todos los colectivos pacifistas» alrededor de una serie de objetivos comunes como fueron el «rechazo a la OTAN, bases y bloques militares, disminución de los gastos militares y [el ser] partidarios de la neutralidad» (García, 1986a). Una Coordinadora constituida por 45 colectivos y que recibió el apoyo de partidos políticos como el MC, la LCR, el PCE o el Partido Comunista de los Pueblos de España (PCPE).

\footnotetext{
${ }^{1}$ La CAO también prestó atención a otros problemas como por el Apartheid surafricano (El País, 1985).
} 
A finales del año 1985 se consigue que el PSOE anuncie la convocatoria del referéndum sobre la permanencia de España en la OTAN incluido en su campaña electoral de 1982, confirmando su «última vuelta de tuerca» discursiva (Andrade, 2007:106).

Un año después, presidida por Antonio Gala, se creó la Plataforma Cívica por la Salida de España de la OTAN, que encuadraba partidos como el PCE — principal instigador-, el Partido de Acción Sindicalista o el Partido Humanista. Un colectivo cuyo único fin era sacar al país de la OTAN y que encerraba claras intenciones de influir en los procesos electorales, lo que chocaría con la CAO, que presentaba un programa más amplio.

La campaña del referéndum fue ardua y constituyó lo que algunos autores han denominado "la última batalla de la transición» (Treglia, 2016: 73). El gobierno y las fuerzas que estaban a favor de la permanencia del país en la OTAN usaron todos los aparatos del Estado para la defensa de sus posiciones, especialmente de los medios de comunicación, lo que dejó a los partidarios del «no» en una manifiesta situación de inferioridad (Martínez Sánchez, 2011: 302). A este hecho habría que sumar la «ingeniería social más avanzada», aplicada por el gobierno a la pregunta de las papeletas del 12 de marzo de 1986, que hizo que, de acuerdo con algunos especialistas, se neutralizaran «los sentimientos antinorteamericanos y antinucleares predominantes en la opinión pública a cambio de su aceptación de la OTAN» (Pastor, 2001: 462). El resultado global de la votación fue el triunfo del "sí» con un 52,50\% de los votos, frente al 38,85\% que apostó por el «no». El esfuerzo empleado en las movilizaciones previas hizo que la izquierda española se desinflara, debiendo reagruparse. El PCE, tras la derrota, puso en marcha la plataforma Izquierda Unida, con la intención de aglutinar al mayor número de partidos a la izquierda del PSOE posibles (Cubero Trujillo, 2016: 66), y fuerzas como el MC o la LCR siguieron profundizando en su estrategia de influir y participar de forma activa en los movimientos sociales, manteniendo su retiro de la vía electoral.

\section{Las radios libres madrileñas}

El movimiento de las radios libres es uno de los grandes desconocidos de la historia de la Transición democrática. Recibió cierta atención a lo largo de la década de 1980, en la que se publicaron una serie de estudios que se pueden considerar clásicos (Prado, 1983 sobre Italia; Aguilera, 1985, una aproximación general; Dolç, Sanchís \& Deó, 1985, sobre Cataluña), durante la década siguiente se publicaron varios trabajos desde disciplinas como las Ciencias de la Información o la Sociología que tenían a estas emisoras por objeto de estudio (Santos Díez 1994, sobre el País Vasco; Martínez 1998, sobre Madrid) y ha sido recientemente cuando se ha comenzado a mostrar interés por el fenómeno desde la historiografía (Pérez 
Martínez, 2018 sobre Madrid y García Gil, Gómez García \& Reguero Sanz, 2018, sobre todo el Estado).

Estas pequeñas emisoras de Frecuencia Modulada (FM) aparecieron durante la década de 1970 en Francia e Italia y desde allí su influencia llegó a España, donde estas radios sin licencia comenzaron a surgir poco a poco. En Madrid podemos encontrar un primer antecedente en el caso de Radio La Voz del Pobre en 1976, aunque hasta 1983 no apareció la primera generación de emisoras libres propiamente dichas: Onda Sur, Onda Verde Vallekana, Radio Luna y Radio Fhortaleza. Es en este mismo año cuando se celebró en el barrio de Villaverde, auspiciado por Onda Sur, el VI Encuentro de la Coordinadora Estatal de Radios Libres, del que salió el Manifiesto de Villaverde, la primera definición de radio libre española.

Un movimiento que nació en el seno de lo que puede considerarse una subcultura o esfera alternativa, un subconjunto social con unas fronteras permeables, con unos significados, representaciones y prácticas culturales compartidas, constituida por militantes de la izquierda — más o menos radical—y activistas de los movimientos sociales, heredera, de alguna forma, de la oposición antifranquista, influenciada por la nueva izquierda europea y que, a pesar de estar conformada por varias culturas políticas, compartía espacios de sociabilidad e intervención y un horizonte altermundista y de crítica antisistema. Su firme propuesta de modos de vida alternativos y su convicción en la necesidad de transformar el sistema político, social y económico en el que se insertaba España - propuesta de nuevos modos de producción, respeto al medio ambiente, generación de nuevas formas y espacios de ocio alejados de las dinámicas consumistas, etcétera - son las realidades sobre las que se construye esta idea de lo alternativo. Feministas, ecologistas, antimilitaristas, antinucleares, «maos», «trostkos», libertarios, según avancen los años ochenta los «autónomos», insumisos, okupas, son los grupos que conformaron dicha alternatividad y fueron aquellos que terminarían formando parte de estas radios.

Las emisoras madrileñas crecieron entre 1984 y 1987, surgiendo en prácticamente todos los barrios de la capital y en muchas de las localidades de alrededor: La Cadena del Water, Radio Vallekas, Radio Rara, Onda Latina, Radio Jabato, etcétera. Estos proyectos fueron ganando importancia dentro del movimiento, asistiendo a los distintos encuentros de la Coordinadora, posicionándose en las distintas luchas y participando en las respuestas ante los ejercicios represivos de las distintas administraciones.

En 1987, ante el incremento de las emisoras libres, la proliferación de radios piratas y la presión de las emisoras comerciales para que se pusiera orden en el éter y se sacaran nuevas licencias a concurso, el ejecutivo socialista aprobó la LOT, texto legal que vino a regular la radiodifusión e incluyó unas medidas que reprimían duramente a las emisoras libres. La ley recogió también la puesta en marcha de un Plan Técnico Nacional que se tradujo en un concurso de frecuencias a celebrar en 1989. 
Pacifismo, antimilitarismo y libertad de expresión en Madrid (1984-1990): Radio Cero, la radio anti-OTAN

La LOT puso al movimiento de las radios libres en alerta. Se realizaron campañas por el reconocimiento de su especificidad, por sus licencias y por la partición del éter para que pudieran sobrevivir los tres sectores de la comunicación — público, privado y social (Barranquero Carretero, 2015: 16) - , todo en medio de un clima de tensión que estaba haciendo que el movimiento de las radios libres en Madrid se dividiese. Aparecieron nuevas emisoras como Radio ELO u OMC Radio, se celebraron encuentros de las emisoras de la Comunidad de Madrid, como el de Rivas Vaciamadrid en 1988, y de la Coordinadora Estatal, todos centrados en el concurso de licencias, y la fractura madrileña se hacía cada vez más evidente alrededor de dos bloques definidos: las futuras radios comunitarias y las radios libres.

El primero de los grupos se articuló en torno a Onda Verde, emisora muy activa durante estos años, y el segundo alrededor de la dupla Radio Cero/Antena Vicálvaro. A estos dos bloques hay que sumar emisoras como La Cadena del Water, que se mantuvieron independientes durante toda la década. Así, al concurso de licencias se presentó, por un lado, la Federación de Radios Libres de la Comunidad de Madrid (FERALICOMA), correspondiente al segundo grupo, y por otro lado la Agrupación de Asociaciones Culturales de Comunicación de la Comunidad Autónoma de Madrid (AACC-CAM), aquel grupo que había articulado Onda Verde, emisora que decidió presentarse finalmente en solitario, al igual que La Cadena del Water.

Una vez resuelto el concurso solamente dos licencias se concedieron al movimiento de las radios libres, una a Radio Klara, en Valencia, que sigue activa hoy en día, y otra a FERALICOMA, en Chinchón, una localidad a 44 kilómetros de Madrid. Esta segunda licencia terminó constituyendo un problema para las emisoras que integraban la Federación. El desgaste al que se vieron sometidas fue considerable y ante la imposibilidad de gestionar el proyecto, el mismo se terminó vendiendo a Ruiz Mateos, empresario conocido por sus múltiples fraudes (Gómez, 2015).

La emisora de Chinchón desapareció en los primeros compases de la década de 1990, años duros para las radios libres madrileñas, que recuperaron su actividad en la de los 2000, momento en el que las emisoras estabilizaron su presencia en el éter, manteniendo las mismas reivindicaciones de los años ochenta.

\section{Radio Cero, la radio anti-OTAN de Madrid. Un poco de historia}

Fue en el mes de octubre de 1984 cuando Radio Cero salió a las ondas madrileñas desde sus céntricos estudios en la calle Rodríguez San Pedro. Sin embargo, el proceso de creación de la emisora había comenzado tiempo antes. En 1983 se suceden una serie de reuniones en las que se debate sobre la necesidad de poner en mar- 
cha una emisora libre desde la CAO y a las que asisten miembros de la Comisión - Carlos Otamendi y Gabriel Flores-, militantes del MC -Ernesto Portuondo y Javier Ortiz-, activistas de la Asociación de Estudios y Protección de la Naturaleza (AEPDEN) - Juan J. Damborenea - e individuales —Alfredo Grimaldos—22.

Parte de este grupo había participado en Radio Luna, emisora libre madrileña relacionada con CNT, y ellos lanzaron la idea de dotar a la CAO de una radio propia, como una extensión de Zona Cero, su revista. De acuerdo con Juan J. Damborenea:

un grupo de gente que estábamos metidos en el movimiento ecologista de aquí de Madrid empezamos a trabajar en una radio que existía, [..] que estaba dentro de los locales de la CNT [...] que se llamaba Radio Luna. [...] Hubo un momento en que allí hubo unos problemas, que no podía estar la radio [episodio ligado a la escisión entre CNT y CGT], etcétera, y entonces un grupo de gente que estábamos ligados al movimiento Anti-OTAN planteamos la posibilidad de hacer una radio que sirviera de plataforma para la Comisión Anti-OTAN³.

Una decisión política que se madura una vez visto el potencial que una emisora podía tener como medio de difusión de la actividad de la CAO dentro de Madrid, dejando entrever que, de hecho, en un primer momento pudo haberse entendido la radio tan solo como un medio para la proyección de la labor de la Comisión:

Para nosotros lo fundamental es la Comisión Anti-OTAN. Como Comisión es un instrumento importante que consideramos que puede fortalecerla, esa cuestión es muy importante a la hora de decidir ese asunto. Y claro, no es, digamos, crear un espacio político, crear una estrategia alternativa o pensar que las radios libres son la hostia y pueden negociar con el gobierno para sacar pelas, de eso no había nada de nada, no. Sino más bien es una proyección de hacer más fuerte el trabajo de la Comisión Anti-OTAN, de que tenga más repercusión, de que llegue a gente nueva, de tener cierto elemento de expresión para poder decir desde ahí también nuestras cosas, sin darle un gran planteamiento político, ni estratégico, ni nada de eso, sino uno de los instrumentos que teníamos, igual que teníamos la revista, pues teníamos la radio, en fin, esas cosas.

\footnotetext{
2 Archivo del Autor. Entrevista a Alfredo Grimaldos, miembro de Radio Cero, cercano al núcleo fundador, y de Radio Carcoma, Madrid, 04/03/2011 y Archivo del Autor. Entrevista a Juan J. Damborenea, miembro fundador de Radio Cero, Madrid, 13/03/2012.

3 Archivo del Autor. Entrevista a Juan J. Damborenea, 13/03/2012.

${ }^{4}$ Archivo del Autor. Entrevista a Gabriel Flores, miembro de la Comisión Anti-OTAN muy implicado en la creación de Radio Cero, Madrid, 12/09/2012.
} 
Una vez tomada la decisión, comienza una campaña de recogida de fondos para conseguir las 100.000 pesetas iniciales a través de donaciones y de bonos de ayuda de la CAO. Se escoge como sede un piso en la calle Rodríguez San Pedro número 2, donde ya se encontraban otras iniciativas vinculadas al $\mathrm{MC}$ y a la $\mathrm{CAO}^{5}$. Un edificio alto y de oficinas, lo que permitía un mayor alcance de las emisiones y una convivencia pacífica con los vecinos en las horas más intempestivas. Un local que, como recordaba José Babiano, que emitió en Radio Cero durante un tiempo: «era un apartamento bien pequeño [...], entrabas a una sala y luego estaba el estudio con la pecera y con la parte técnica» ${ }^{6}$.

Prácticamente desde el comienzo la emisora vivió bajo la acusación — por parte de un sector de las radios libres- de ser el altavoz del MC, lo que desató múltiples polémicas a lo largo de los años ${ }^{7}$. Los testimonios de los protagonistas inciden en una misma dirección, pese a su vinculación con la CAO de Madrid la emisora siempre actuó como un ente independiente a todos los niveles. Ernesto Portuondo, miembro fundador y muy activo en la radio, recordaba que:

[...] Radio Cero sí nace con una estrecha vinculación a la Comisión Anti-OTAN, ahora bien, su funcionamiento y todas las decisiones que se han tomado en Radio Cero [...], era [a través de] un método asambleario [...]. Había un colectivo [...] de la coordinadora o no me acuerdo cómo se llamaban, que se encargaba de mantener más o menos las decisiones, y en ese colectivo el MC si acaso tendría una o dos personas como mucho, entre ocho, nueve o diez ${ }^{8}$.

José Manuel Roca, miembro de la emisora, es claro también a este respecto cuando reconoce que, desde su punto de vista, la radio:

[...] ni el propio MC la utilizaba como plataforma [...], de vez en cuando llegaba allí alguien para una entrevista, [...] pero desde la asamblea que dirigía aquello yo nunca observé que dependiéramos del $\mathrm{MC}^{9}$.

${ }^{5}$ La agencia de viajes Años luz, perteneciente a Daniel Wagman, militante del MC y la CAO (Garí, 2012).

6 Archivo del Autor. Entrevista a José Babiano, miembro de Radio Cero, Madrid, 04/02/2011.

7 Hay que reconocer que el MC sí mostró interés por las radios libres, publicando múltiples artículos en su periódico, Servir al pueblo (Informe Radios Libres, 1984 y Ortiz, 1984, p. 13), y llegando a tener un "activo» (célula sectorial) dedicado a este movimiento. Una de las polémicas más importantes surgió cuando Esteban Ibarra, coordinador de Onda Verde, filtró un documento de dicho «activo» en base al cual se acusó duramente a emisoras como Radio Cero de ser radios de partido (La Alcarria Obrera, 2010).

8 Archivo del Autor. Entrevista a Ernesto Portuondo, miembro fundador de Radio Cero, Madrid, 09/05/2008.

9 Archivo del Autor. Entrevista a José Manuel Roca, miembro de Radio Cero, Madrid, 03/01/2012. 
Parece que la independencia, tanto en lo orgánico como en lo financiero, era total, lo que no quita para que de alguna forma fuera beneficioso tener cerca a la militancia de aquel partido ya que:

$[\ldots]$ a la gente que estábamos allí de sectores independientes [...] nunca se nos impuso nada, lo que pasa es que a nosotros nos hacía la vida más sencilla porque era una organización que tenía un entramado muy fuerte y entonces claro, para nosotros, se decía: "se necesita un electricista» y entonces, «pues conocemos a uno que trabaja en una fábrica en no sé qué» y te venía y te organizaba todo el tendido de luces. Entonces en ese sentido... lejos de hacernos daño yo que creo solo hizo beneficiarnos. [...] sin el MC la radio nunca habría funcionado, absolutamente imposible. [...]. Pero allí no se pedía a nadie ningún tipo de carné ni de nada para entrar ${ }^{10}$.

La emisora comenzó su andadura con unos compromisos claros: evitar el «rollo doctrinal o mitinero», y «dar informaciones y opiniones sencillas, cortas y claras, sin ocultar nuestro punto de vista comprometido». Todo esto con el objetivo de «ir más allá de los convencidos y los enterados», de hacer una radio para todo el mundo, buscando la mayor participación posible: «dando un papel relevante a las llamadas telefónicas de los oyentes» y abriendo los espacios informativos «a un espectro plural y diverso, siempre dentro del campo de la izquierda»( Antonio \& Ernesto, 1984: 6 y 7).

El tiempo de emisión de Radio Cero fue creciendo con el paso de los años. De una programación de lunes a viernes de 9 de la noche a 1 de la madrugada, y los sábados y domingos de 7 de la tarde a 10 de la noche, se pasó a otra que entre semana iría de las 6 de la tarde a las 2 de la madrugada, para terminar, tras la mejora del aparataje técnico de la emisora, emitiendo 24 horas diarias ${ }^{11}$.

En cuanto a sus contenidos, se mantenía un paralelismo con el resto de los proyectos de comunicación alternativa de Madrid. Era normal, por lo tanto, encontrar programas musicales de distintos estilos y espacios dedicados a los distintos movimientos sociales ${ }^{12}$. De entre estos podemos destacar «Libertad condicional», realizado por miembros del Comité Antirrepresivo de Madrid ${ }^{13}$; un programa antimilitarista dirigido por «El Peque», militante de la LCR ${ }^{14}$; o «Y el verbo se hizo carne»,

\footnotetext{
10 Archivo del Autor. Entrevista a Juan J. Damborenea, 13/03/2012.

11 Archivo del Autor. Entrevista a Ernesto Portuondo, 09/05/2008.

12 Archivo del Autor. Entrevista a Ernesto Portuondo, 09/05/2008.

13 Archivo del Autor. Entrevista a Juan Pablo Calero, miembro de Radio Cero, Alcalá de Henares, $16 / 12 / 2010$.

14 Archivo del Autor. Entrevista a Juan J. Damborenea, 13/03/2012.
} 
programa anticlerical dirigido por una militante del $\mathrm{MC}^{15}$, entre otros. Radio Cero emitió también un espacio contrainformativo - formato clásico de este tipo de emisoras- con el nombre de "Como lo oyes».

La emisora sufrió problemas como cortes del cableado de la antena e interrupción de su señal en momentos en los que los contenidos que se estaban radiando podían resultar comprometedores. Un hecho que hizo a sus miembros asumir que, de alguna manera, las autoridades controlaban su actividad. En consecuencia, se tomaron una serie de medidas de seguridad que despertaron cierta hilaridad entre los miembros de la radio:

había una cosa que decía "Que no abráis la puerta sin mirar por la mirilla, si viene la policía encerraros y resistir [sic.], estos son los teléfonos del abogado, solo por la mañana» o "solo tal hora a tal hora" vamos que $[\ldots]$ venían las horas a las cuales podías llamar al abogado. ¿Y el resto del tiempo que haces? ¿Resistimos aquí? "Espere un momento que no podemos llamar al abogado, que aún no ha venido, que esta con los niños»... ${ }^{16}$.

Dejando a un lado estos problemas, Radio Cero se convirtió durante la década de 1980 en un referente para las radios madrileñas. Alineada con Antena Vicálvaro sostuvo un enfrentamiento con Onda Verde y colectivos afines, en el que confrontaron dos modelos distintos de entender la radiodifusión alternativa. La emisora estuvo muy activa en la campaña que se inició tras la aprobación de la LOT y, de cara al concurso de licencias de 1989, una vez se manifestó que el movimiento en Madrid se había fracturado, fue una de las emisoras que conformó FERALICOMA, resultando agraciada con una.

La emisora de Chinchón se convirtió en prioritaria para aquellos proyectos que conformaban la Federación, lo que hizo que Radio Cero decidiera cesar su actividad en 1990, entendemos que porque volcaron sus esfuerzos en la nueva emisora legal. Con la desaparición del proyecto de Chinchón, y la posterior disolución de FERALICOMA, el espíritu de la emisora que aquí nos ocupa terminó por desvanecerse, tal vez con las ondas de su última emisión.

15 Archivo del Autor. Entrevista a Alfredo Grimaldos, 04/03/2011.

16 Archivo del Autor. Entrevista a Juan Pablo Calero, 16/12/2010. 


\section{Libertad de expresión, pacifismo y antimilitarismo en el día a día de Radio Cero}

¿De qué forma implementó la emisora en su praxis cotidiana las reivindicaciones a las señaladas más arriba? Acercarse a la Declaración fundacional de la emisora, documento con siete puntos, constituye el primer paso para entender qué movió al colectivo y qué importancia tuvieron el antimilitarismo y el pacifismo dentro su estructura. Así, entre los objetivos de la emisora se encontraban:

1. Servir de medio de expresión y comunicación al conjunto de los movimientos sociales, culturales y políticos que se enfrentan al sistema de poder en los distintos terrenos, con el objetivo de hacer una sociedad más libre, más igualitaria, justa y placentera.

1.1. Especialmente nos sentimos vinculados al movimiento pacifista y antimilitarista que expresa su oposición a la OTAN, a las bases norteamericanas, a la política de bloques y de alineamiento militar, al armamentismo, al servicio militar obligatorio y a la discriminación o represión contra los jóvenes que se niegan a cumplirlo. [...]

1.7. A todos aquellos movimientos, asociaciones e iniciativas que defiendan las libertades individuales y colectivas contra las arbitrariedades del poder, el autoritarismo de los estados y la brutalidad de los ejércitos. $[\ldots]^{17}$

Vemos cómo dentro de la defensa y apoyo a los movimientos sociales - el documento hace mención también a otros movimientos como el feminismo o el ecologismo- la lucha contra la OTAN, como uno de los ejes de la lucha antimilitarista y pacifista, ocupa un lugar preeminente en el ideario de la emisora. Una proyección y defensa de los principios del antimilitarismo que camina pareja al desarrollo de los principios que guiaban la práctica del movimiento de las radios libres. De hecho, los puntos del dos al siete de la Declaración apuntan en la misma dirección que aquellos contenidos en el Manifiesto de Villaverde, documento que desde 1983 definía qué era una radio libre en España ${ }^{18}$ : no profesionalización, actividad sin ánimo de lucro, comunicación horizontal y abierta, organización participativa, etcétera. La defensa y apoyo de los movimientos sociales propuesta por Radio Cero funciona en esta misma dirección y se corresponde con la vocación última de estas emisoras de «dar voz a los sin voz».

17 Archivo del autor. Radio Cero: Radio Cero: Programación, Madrid, 1988, fotocopia de tríptico original.

18 Archivo del autor. Coordinadora Estatal de Radios Libres: Manifiesto de Villaverde, Madrid, 1983, fotocopia de original. 
Este apoyo a la lucha pacifista/antimilitarista se hizo, por un lado, a través de la puesta en marcha de programas específicos. Uno de estos espacios desde los que se dio cobertura a las movilizaciones pacifistas, con una preeminencia de la evolución de la lucha anti-OTAN, fue el magacín "Esto es Cero», realizado cada día, durante unos años, por «un equipo diferente y especializado» en las más diversas temáticas: «libertad-represión-marginación social, mundo sindical y laboral-paro, luchas de las mujeres, movimiento ecologista y movimiento por la paz» (Antonio \& Ernesto, 1984: 6 y 7). Por otro, dando proyección y cobertura a los distintos actos y manifestaciones de la lucha contra la OTAN, llegando incluso a informar de ellos en directo: era costumbre de varios de los programas de la parrilla de Radio Cero el seguimiento de manifestaciones vía telefónica. Una cobertura precaria en lo técnico —al tratarse de llamadas desde teléfonos públicos- que, no obstante, tuvo su importancia ya que por su inmediatez superaba las vías de información generalistas. La posibilidad de atender a la evolución de una manifestación con actualizaciones más o menos en directo era una realidad hasta ese momento impensable, además de ahondar, en muchas ocasiones, en aquel feedback y en aquella comunicación horizontal que buscaban este tipo de emisoras. Una actividad que alcanzó tal intensidad que Gonzalo Casanova valora que:

los meses previos al referéndum de 1986 sobre la integración española en la OTAN fueron el primer banco de pruebas relevante en el que el conjunto de radios libres sirvieron para crear debate y animar la campaña anti-OTAN ofreciendo una amplia contrainformación o información alternativa a la oficial. Por primera vez, se valora que sólo las radios libres pueden cubrir «en caliente» la más rabiosa actualidad, contribuyendo a generar bloques sociales amplios de oposición a las políticas oficiales. (Casanova, 2002: 19)

Informar sobre las distintas campañas, dar los puntos de vista de la CAO, generar debate alrededor de la actualidad del movimiento, etcétera, constituyen una consecución de aquellos postulados de las radios libres vinculados con la libertad de expresión a través de la libertad de emisión por la vía de la praxis. La ruptura con el control mediático e informativo de las clases dominantes para concienciar de una causa como la anti-OTAN, permitiendo a los ciudadanos participar activamente en la generación de contenidos e información, era, de facto, una vía de participación y de creación de discurso contrahegemónico. Y era esta participación en los procesos comunicativos y en la defensa de las distintas movilizaciones lo que buscaban aquellas personas que pusieron en marcha la emisora. De acuerdo con Gabriel Flores:

[...] ahí aprendíamos también de cómo se trabaja con gente que no coincide contigo en muchísimas cosas. [...] Para nosotros ese rollo de las radios libres era una cosita minúscula dentro de todo el panorama, [...] nosotros estábamos en otra completamente diferente que era fortalecer, ampliar, vincu- 
larnos a nueva gente, tener movimientos de presión, experimentar, todo ese rollo. [...] nosotros lo que queríamos es que la radio funcionase lo mejor posible, que la gente que estuviera ahí estuviera haciendo su trabajo, que si podía aportar algo lo aportase, que si nosotros podíamos aportar algo, lo aportásemos y ese es nuestro rollo ${ }^{19}$.

Este testimonio refleja dos realidades. Por un lado, la de la participación en el proyecto radiofónico de todas aquellas personas interesadas, que sería clave para la superación de la comunicación tradicional y, por otro, las relaciones que se establecieron dentro de la propia radio y entre las personas y grupos de su entorno, como el MC. Esta relación, en términos de aprendizaje mutuo y colectivo, sería una de las potencialidades de las emisoras libres para la proyección de luchas sociales.

Como ya se ha apuntado para otros movimientos sociales como el feminismo (Pérez Martínez, 2013), las emisoras libres constituyeron instancias de producción y reproducción ideológica. Al convertirse en un «movimiento de movimientos», un espacio en el que participaban libremente personas y colectivos provenientes de distintas luchas, los discursos de estas se retroalimentaban y extendían su influencia en diferentes direcciones. En primer lugar, estas ideas se ponían en común dentro de la estructura de las propias emisoras. En el caso de Radio Cero y la movilización contra la OTAN, las personas que se fueron incorporando al proyecto entraron en contacto con las posiciones de la $\mathrm{CAO}$, conociéndolas y pudiendo asumirlas como propias, incorporándose a la misma, o generando debate alrededor de ellas. Así, aquellos provenientes de otros movimientos sociales, o que se sumaran al proyecto por su interés en lo musical o lo comunicativo, conectarían, en Radio Cero, con el pacifismo y el antimilitarismo, facilitando así su concienciación.

En un segundo lugar, y teniendo en cuenta ese rol de la radio de aparato ideológico (Althusser, 1988), Radio Cero habría proyectado los discursos de la CAO y su defensa del pacifismo y el antimilitarismo a dos niveles. El primero de ellos, el más inmediato, fue a esa esfera alternativa en la que vimos se insertaban este tipo de emisoras, y cuya escucha constituía una práctica cultural definitoria de la misma. La sintonización de la programación de Radio Cero hizo llegar a los miembros de este subconjunto social sus posiciones dentro de la diversidad que encerraba el movimiento pacifista, con el mismo efecto que aquel detectado en la puesta en común de dicho discurso dentro de la estructura de la radio. Los participantes en dicha esfera que proviniesen de diferentes contextos — distintos partidos políticos, otros movimientos sociales, otras ramas del pacifismo, etcétera- confrontarían en las emisiones de Radio Cero con los puntos de vista de la CAO.

19 Archivo del Autor. Entrevista a Gabriel Flores, 12/09/2012. 
El segundo de los niveles fue dado por la capacidad de las ondas hertzianas para superar fronteras, en este caso los porosos límites de ese espacio alternativo, y llegar al resto de la sociedad. En este caso concreto Radio Cero intentó convertirse en un fenómeno metropolitano (Carmona, 1987: 15), es decir, intentó alcanzar con sus emisiones a toda la ciudad de Madrid, con lo que se puede deducir que sus ondas llegarían a personas ajenas a ese entorno natural de la emisora, a ese ecosistema alternativo. Es difícil valorar el impacto ideológico que pudieron tener los discursos emitidos por Radio Cero en la audiencia madrileña, pero parece que el total de las emisoras libres pudieron haber llegado a plantear una contrahegemonía que hizo que, de cara a ganar el referéndum de 1986, el gobierno tuviese que aplicarse a fondo.

Debe valorarse también cómo en las dos primeras modalidades de difusión, dentro de la emisora y dentro de la esfera alternativa, las dinámicas de retroalimentación de las radios libres pudieron favorecer el enriquecimiento de dichos discursos. El funcionamiento asambleario de Radio Cero pudo dar lugar a debates alrededor de las posiciones de la CAO y la participación de la audiencia a través del teléfono pudo matizar las opiniones vertidas desde la radio. Finalmente, la apertura del colectivo permitió a aquellas personas interesadas en realizar un programa sumarse al mismo, incorporando sus propias opiniones al discurso de la emisora. En definitiva, en el seno de Radio Cero se generó un proceso de construcción discursiva participativo en el que las posiciones de la CAO bebieron de las aportaciones de sus participantes — en la asamblea u oyentes- Se unieron, en base a estos mecanismos de elaboración y proyección ideológica, en el devenir cotidiano de la emisora, la lucha pacifista y el ejercicio de la libertad de expresión.

Estos ciclos de circulación ideológica facilitaron la creación de una «esfera pública periférica» (EPP) que terminaría por instaurarse en "contrahegemonía». Dentro del subconjunto social que constituye la subcultura alternativa, y a través del proceso descrito anteriormente, se desarrolló una EPP, diferente de la esfera pública central, dependiente de los grupos dominantes y producto de la hegemonía social ejercida por los mismos. Una EPP que se caracterizó por desplegar en su estructura «autogestión, coparticipación y prácticas colaborativas o de intercambio», por arrojar «un mayor pluralismo de voces, tendencias ideológicas y diversidad de agenda» y por la interacción de su público, inmerso «en un proceso constante de deliberación» (Resina \& Sampedro, 2010: 151). En este ambiente es dónde se fragua una hegemonía alternativa, es decir, dentro de este subconjunto social las propuestas ideológicas y culturales en circulación se convierten en «dominantes» y contestan la hegemonía social, en tanto que formación cultural emergente. Concretando un poco más, la cohesión obtenida a nivel cultural por el grupo alternativo lo convertiría en un sitio de resistencia frente a la cultura dominante y entraría a formar parte de los procesos de negociación de la hegemonía social.

Es en este punto donde entra en juego la proyección de contenidos ideológicos hacia fuera de los límites de la esfera alternativa. A través de instituciones, como 
las emisoras libres, y del contacto humano en espacios de sociabilidad comunes, los planteamientos de la subcultura alternativa transcendieron sus fronteras. Igualmente llegaron a la esfera del grupo dominante por su progresiva expansión, de tal manera que la ideología de la subcultura y su praxis tuvo que ser tomada en consideración por éste para no perder su hegemonía. De cara a no ver peligrar su posición, la clase dominante incorporó parte de la agenda y la praxis de la esfera alternativa a su propio repertorio cultural de manera que, aunque perdieron su componente subrepticio, estas incorporaciones pasaron a formar parte de la cultura dominante y entraron de nuevo en los flujos de circulación ideológica.

Es en este momento en el que puede reconocerse el «relativo» éxito de las radios libres en el contexto general de la sociedad. Por la propia naturaleza de este tipo de medios de comunicación no se dispone de datos estadísticos fiables sobre sus niveles de audiencia ${ }^{20}$. Sin embargo, el impacto mediático que tuvieron, con apariciones constantes en medios impresos generalistas de distinto tipo — desde diarios como El País, ABC o La Vanguardia, a revistas como Fotogramas (Luzán \& Aymerich, 1978: 10-12), Diario 16 (Antona \& Regueiro, 1984: 4-14) o Blanco y Negro (Criado, 1980: 27-30)- y una importante cobertura en revistas y periódicos alternativos - Ajoblanco, La puça y el general, El Viejo Topo e incluso Mundo Obrero-, es una representación significativa del peso que tuvieron en la sociedad española del momento, formando parte de su agenda política y social.

En base a este calado social, entendemos que, a través de su labor y su papel como agentes mediadores en la negociación de la hegemonía social del momento, se incorporaron a la cultura dominante tanto contenidos como valores, o formas de actuar de la subcultura alternativa. La preocupación por el ecologismo, avances en el feminismo y el auge del antimilitarismo y el pacifismo, así como otros valores, no entraron a formar parte del acervo cultural de la España de aquellos antes sin que previamente hubieran formado parte de una hegemonía alternativa y se hubiesen extendido, tanto horizontal como verticalmente, por toda la sociedad.

\section{Conclusiones}

La experiencia de la madrileña Radio Cero, la radio anti-OTAN, puede entenderse como paradigmática de las relaciones que establecieron los distintos movimientos sociales con el de las radios libres durante la década de los ochenta. Una re-

${ }^{20} \mathrm{Ha}$ de tenerse en cuenta que para aparecer en el principal medidor de audiencias, el Estudio General de Medios hay que pagar. Al no perseguir el lucro, las radios libres no aparecían recogidas en dichas encuestas. 
lación que plantea un modelo hipotético de expansión de los ideales de los distintos movimientos sociales en la sociedad española del momento. Esta emisora, con su especial vocación en la lucha contra la OTAN, ayudó, por lo tanto, a la proyección y apoyo de esta movilización y de los valores que la misma conllevaba, matizados siempre por los planteamientos de la $\mathrm{CAO}$, colectivo que se encontraba, en un origen, detrás de la misma.

Profundizar en este tipo de estudios permite mejorar el conocimiento sobre cómo se relacionaron los diferentes agentes que conformaron la subcultura alternativa, esa esfera en la que las radios libres jugaron un importante papel ideológico. Su participación en la generación de una «esfera pública periférica» que se tornó en contrahegemónica, constituyó una de sus funciones más importantes. Radio Cero, a través de su praxis cotidiana, habría enlazado perfectamente la lucha por la libertad de expresión y la libertad de emisión, con el pacifismo y las reivindicaciones de otros movimientos, haciendo de altavoz, de amplificador, a los mismos, permitiéndoles aumentar su alcance social.

Hay que profundizar, a pesar de la escasez documental y de lo complicado de medir las audiencias de estos medios, en este tipo de estudios de cara a poder seguir recuperando el importante papel jugado por las radios libres y el total de los movimientos sociales en los años de la Transición y una vez se conformó esta, cómo crecieron, cómo se desarrollaron y, sobre todo, de qué forma se relacionaron entre ellos e incidieron en la sociedad española.

\section{Fuentes}

Archivo del autor. Coordinadora Estatal de Radios Libres: Manifiesto de Villaverde, Madrid, 1983, fotocopia de original.

Archivo del autor. Entrevista a Alfredo Grimaldos, miembro de Radio Cero, cercano al núcleo fundador, y de Radio Carcoma, Madrid, 04/03/2011.

Archivo del Autor. Entrevista a Ernesto Portuondo, miembro fundador de Radio Cero, Madrid, 09/05/2008.

Archivo del Autor. Entrevista a Gabriel Flores, miembro de la Comisión Anti-OTAN muy implicado en la creación de Radio Cero, Madrid, 12/09/2012.

Archivo del Autor. Entrevista a José Babiano, miembro de Radio Cero, Madrid, 04/02/2011.

Archivo del Autor. Entrevista a José Manuel Roca, miembro de Radio Cero, Madrid, 03/01/2012.

Archivo del Autor. Entrevista a Juan J. Damborenea, miembro fundador de Radio Cero, Madrid, 13/03/2012. 
Archivo del Autor. Entrevista a Juan Pablo Calero, miembro de Radio Cero, Alcalá de Henares, 16/12/2010.

Archivo del autor. Radio Cero: Radio Cero: Programación, Madrid, 1988, fotocopia de tríptico original.

\section{Referencias bibliográficas}

«Informe: Las radios libres», Servir al pueblo, 23 de marzo al 5 de abril, 1984, pp. 11-14.

Aguilera, M. (1985). Radios libres y radios piratas. Madrid: Forja.

Aldecoa, F. (1989). Las constantes de la política exterior española. Política y sociedad, 2, 61-78.

Althusser, L. (1988). Ideología y aparatos ideológicos del Estado. Freud y Lacan. Buenos Aires: Nueva Visión.

Andrade Blanco, J.A. (2007). Del socialismo autogestionario a la OTAN: notas sobre el cambio ideológico en el PSOE durante la transición a la democracia, Historia actual on-line, 14, 97-106.

Antona, M. y Regueiro, A. (1984). Las otras radios, solas y libres en la madrugada. Diario 16, $139,4-14$.

Antonio y Ernesto (1984). Radio Cero. Lo que faltaba», Zona Cero, 6, 1984, pp. 6 y 7.

Barranquero Carretero, A. (2015). Definición y rasgos del tercer sector de la comunicación. En A. Barranquero Carretero (coord.), Juventud española y los medios del Tercer Sector de la Comunicación (pp. 16 y 17). Madrid: Centro Reina Sofia.

Carmona, M. (1987). Las radios libres. Cuaderno Gris 1, 14-16.

Casanova, G. (2002). Armarse sobre las ruinas. Historia del movimiento autónomo en Madrid (1985-1989). Madrid: Potencial.

Criado, A. (1980). Radios libres. La guerrilla de la información. Blanco y negro, 3532, 27-30.

Cubero Trujillo, I. M. (2016). CUBERO TRUJILLO, Isabel $\mathrm{M}^{\mathrm{a}}$, «El proceso de integración de España en la OTAN y la reconfiguración social y política de la izquierda», Historia actual on-line, 41, 3, 57-69.

Dolç, M., Sanchis, V. y Deó, F. J. (1985). Les ràdios lliures: una pràctica alternativa, Terra Verda, Barcelona, 1985.

El País (1985, agosto 20). La comisión anti-OTAN. El País. Recuperado de https://elpais. com/diario/1985/09/06/internacional/494805602_850215.html.

El País (1986, marzo 3). Cientos de miles de personas participaron en las manifestaciones contra la Alianza Atlántica. El País. Recuperado de https://elpais.com/ diario/1986/03/03/espana/510188417_850215.html

Fernández Amador, M. y Vidal Gómez, A. (2004). El debate sobre la OTAN entre los socialistas de Almería. En C. Navajas Zubeldía (coord.). Actas del IV Simposio de Historia Actual (pp. 929-944). Instituto de Estudios Riojanos, Logroño, 2004. 
García, R. (1985, marzo 25). Decenas de millares de personas participaron en la marcha contra la base de Torrejón y por la salida de la OTAN. El País. Recuperado de: https:// elpais.com/diario/1985/03/25/espana/480553212_850215.html

García, R. (1986b, marzo 3). Intelectuales, artistas y pacifistas sustituyen a los políticos en la cabeza de las manifestaciones anti-OTAN. El País. Recuperado de: https://elpais. com/diario/1986/03/03/espana/510188415_850215.html

García, R. (1986a, abril 5). La Comisión Anti-OTAN convoca una nueva marcha a Torrejón. El País. Recuperado de: https://elpais.com/diario/1986/04/05/ espana/513036013_850215.html

García Gil, S., Gómez García, S. y Reguero Sanz, I. (2018). Espacios alternativos de libertad durante la Transición. Breve historia de las radios libres en España (1976-1983). Revista latina de comunicación social, 73, 10, 179-1210.

Garí, M. (2012). In memoriam Daniel Wagman. Good bye friend Dany. Anticapitalistas, Recuperado de: http://www.anticapitalistas.org/spip.php?article26330

Gómez, L. (2015, septiembre 8). La vida piramidal de un empresario milagro. El País. Recuperado de: https://elpais.com/economia/2015/09/07/actualidad/1441619615_713810. html

Gonzalo, C. (2011). El movimiento vecinal español frente a la OTAN: el caso de Valladolid. Historia 396, 2, 247-263.

La Alcarria Obrera (2010). Las radios libres en España, Recuperdo de: https://laalcarriaobrera. blogspot.com/2010/07/las-radios-libres-en-espana.html

Luzán, J. y Aymerich, P. (1978). Ponga una radio libre en cada esquina. Fotogramas, 1622, diciembre, 10-12.

Martínez, M. (1998). Islas de autogestión en un mar de contradicciones. Cuatro movimientos alternativos en el estado español (1978-1998). Recuperado de: http://www.miguelangelmartinez. net/IMG/pdf/1998_movimientos_sociales_Gijon.pdf

Martínez Sánchez, J. A. (2011). El referéndum sobre la permanencia de España en la OTAN. UNISCI Discussion Papers, 26, 283-310.

Mateos, A. (2013). Presentación: la batalla de la OTAN en España. Un tardío ajuste ideológico. Ayer, 103, 3, 13-17.

Mercado, F. (1982, agosto 26). 10.000 personas marcharon hasta Torrejón contra la OTAN. El País. Recuperado de: https://elpais.com/diario/1982/06/07/ espana/392248814_850215.html

Mesa, R. (1982). La política exterior en la España democrática», Revista de Estudios Internacionales, 3, 7-67.

Ministerio de Asuntos Exteriores (2017) España en la OTAN. Recuperado de: http:// www.exteriores.gob.es/RepresentacionesPermanentes/OTAN/es/quees2/Paginas/ Espa\%C3\%B1aOTAN.aspx

Oliver Olmo, P. (2011). El movimiento pacifista en la transición democrática española. En R. Quirosa-Cheyrouza y Muñoz (ed.). La sociedad española en la transición. Los movimientos sociales en el proceso democratizador, (pp. 271-286). Madrid: Biblioteca Nueva. 
Ortiz, J. (1984, marzo 23 a abril 5). Mojarse. Servir al pueblo, 13.

Pastor, J. (2001) El movimiento pacifista (1977-1997). En M. Ortiz Heras (eds.). Movimientos sociales y estado en la España contemporánea, (pp. 457-472). Cuenca: Ediciones de la Universidad de Castilla-La Mancha.

Pérez Martínez, J.E. (2013). Mujeres en la radio libre española (de 1976 a nuestros días). En R. M. Capel (ed.): Presencia y visibilidad de las mujeres: recuperando historia, (pp. 359384). Madrid: Abada Editores.

Pérez Martínez, J.E. (2018). La radio libre en Madrid (1976-1989): los orígenes del movimiento por la libertad de emisión. Commons. Revista de Comunicación y Ciudadanía Digital, 7 (1), 112-143.

Prado, E. (1983). Las radios libres. Barcelona: Mitre.

Powell, C. (2003). España en Europa: de 1945 a nuestros días. Ayer, 49, 81-119.

Resina de la Fuente, J. y Sampedro Blanco, V.(2010). Opinión pública y democracia deliberativa en la Sociedad Red. Ayer, 80, 139-162.

Santos Díez M.T. (1984). Radios comerciales-ondas libres. Bilbao: Onda Cero Radio.

Socorro Arencibia, P. (2015). La última batalla de la Transición: las organizaciones del movimiento anti-OTAN. Trabajo de fin de máster. Oviedo: Universidad de Oviedo, Oviedo.

Treglia, E. (2016). La última batalla de la transición, la primera de la democracia. La oposición a la OTAN y las transformaciones del PCE (1981-1986). Ayer, 103, 71-96.

Wilhelmi Casanova, G. (2001). El movimiento por la paz en Madrid, de la Transición al primer gobierno socialista. Recuperado de: http://historiadelpresente.es/sites/default/ files/congresos/pdf/41/ecoysociedad/Wilhelmi.pdf 